\title{
Galcanezumab for the Management of Migraine: A Systematic Review and Meta-Analysis of Randomized Placebo-Controlled Trials
}

\author{
Ahmed Abu-Zaid ${ }^{1}$, Saud K. AlBatati ${ }^{1}$, Abdullah M. AlHossan ${ }^{2}$, Rayan A. AlMatrody ${ }^{1}$, Ayman AlGzi ${ }^{1}$, \\ Rayan A. Al-Sharief ${ }^{3}$, Faris M. Alsobyani ${ }^{4}$, Amena F. Almubarak ${ }^{5}$, Nadeen S. Alatiyah ${ }^{1}$ \\ 1. Internal Medicine, College of Medicine, Alfaisal University, Riyadh, SAU 2. Surgery, College of Medicine, Alfaisal \\ University, Riyadh, SAU 3. Neurology, College of Medicine, King Abdulaziz University, Rabigh, SAU 4. Internal \\ Medicine, College of Medicine, Arabian Gulf University, Manama, BHR 5. Internal Medicine, College of Medicine, Dar \\ Al Uloom University, Riyadh, SAU
}

Corresponding author: Ahmed Abu-Zaid, aabuzaid@live.com

\section{Abstract}

\section{Introduction}

Migraine is a frequent neurological condition manifested by several episodes of headache. Calcitonin generelated peptide (CGRP) has been shown to play a key role in the pathophysiology of migraine. Galcanezumab is a monoclonal antibody that binds CGRP and inhibits its action, without affecting the CGRP receptor. The aim of this study is to carry out a systematic review and meta-analysis of all randomized placebo-controlled trials that evaluated the efficacy of galcanezumab (120 mg or $240 \mathrm{mg}$ ) for the management of migraine.

\section{Methods}

We screened four databases (PubMed, SCOPUS, Embase, and Cochrane Central) from inception to October 10, 2020. Studies meeting the following criteria were included: (i) Patients: individuals with migraine, (ii) Intervention: galcanezumab at a dose of $120 \mathrm{mg}$ or $240 \mathrm{mg}$, (iii) Comparator: placebo, (iv) Outcomes: prespecified efficacy and safety outcomes, and (v) Study design: randomized placebo-controlled trials. Efficacy outcomes included change in migraine headache days (MHDs), change in MHDs with acute medication use, patient global impression of severity (PGI-S) score, migraine-specific quality of life role function-restrictive domain (MSQ RF-R) score, and migraine disability assessment (MIDAS) score. Safety outcomes included frequency of injection-site pain, nasopharyngitis, and upper respiratory tract infection (URTI). Moreover, we used the Cochrane Collaboration's risk of bias tool to assess the risk of bias of the included studies. Review Manager Software, version 5.4.1, was used for statistical analysis. Mean difference and risk ratio with $95 \%$ confidence interval were used to analyze continuous and dichotomous outcomes, respectively. We used the fixed-effects and random-effects models to analyze homogeneous and heterogeneous data, respectively.

Review began 10/17/2020 Review ended 11/11/2020 Published 11/22/2020

(๑) Copyright 2020 Abu-Zaid et al. This is an open access article distributed under the terms of the Creative Commons Attribution License CC-BY 4.0., which permits unrestricted use, distribution, and reproduction in any medium, provided the original author and source are credited.

\section{Results}

A total of six studies comprising 4,023 patients were included in this systematic review and meta-analysis. When compared to placebo, both doses of galcanezumab were highly effective in decreasing MHDs $(p<0.001)$, reducing MHDs with acute medication use $(p<0.001)$, and improving the PGI-S score $(p<0.001)$. On the other hand, MSQ RF-R and MIDAS scores were significantly enhanced only in the 240-mg dose group $(\mathrm{p}<0.001)$. With regard to side effects, the rates of injection-site pain and nasopharyngitis did not substantially differ between galcanezumab (inclusive of $120 \mathrm{mg}$ and $240 \mathrm{mg}$ ) and placebo groups. Nonetheless, when compared to placebo, galcanezumab $120 \mathrm{mg}$, but not galcanezumab $240 \mathrm{mg}$, substantially correlated with a higher rate of URTI.

\section{Conclusions}

Galcanezumab is clinically safe and efficient for the management of migraine, and the use of a higher dose increases its efficacy. Future research directions should be geared toward determining the optimal dose of galcanezumab in the management of patients with migraine. Moreover, head-to-head comparative studies between galcanezumab and other related anti-CGRP receptor monoclonal antibodies are warranted.

Categories: Neurology

Keywords: calcitonin gene-related peptide, galcanezumab, migraine, headache

\section{Introduction}

Migraine is a frequent neurological condition manifested by several episodes of headache. These episodes are often accompanied by nausea, vomiting, and light hypersensitivity [1]. Migraine is categorized into two main types in accordance with the frequency of headaches: episodic migraine ( $<15$ headache days per 
month) and chronic migraine ( $\geqslant 15$ headache days per month) [2]. Migraine pathophysiology is not exactly known [1]. However, the accumulating body of research highlights a key role of calcitonin gene-related peptide (CGRP) in migraine pathophysiology [3,4]. This notion is supported by the observation that intravenous injection of CGRP results in spontaneous episodes of headache and migraine in migraineurs [3]. Moreover, blood levels of CGRPs are dramatically increased during migraine attacks [4].

Galcanezumab is a monoclonal antibody that binds CGRP and inhibits its action, without affecting the CGRP receptor [5,6]. Many clinical trials were performed investigating the efficacy of galcanezumab for the management of migraine. However, these clinical trials varied substantially with regard to the range of doses used. Moreover, till now, the proposed evidence from these clinical trials is contradictory. Therefore, the need for a comprehensive research that pools this evidence has become more required, which constituted the basic core of why we aimed to conduct this study to fill the literature gap. The objective of this study is to carry out a systematic review and meta-analysis of all randomized placebo-controlled trials that specifically evaluated the efficacy and safety of galcanezumab (120 mg or $240 \mathrm{mg}$ ) in patients with migraine.

\section{Materials And Methods Research protocol}

This research was conducted in compliance with the Preferred Reporting Items for Systematic Reviews and Meta-Analyses (PRISMA) guidelines [7] and the Cochrane Handbook for Systematic Reviews of Interventions, Version 5.1.0 [8].

\section{Search strategy}

Four databases (PubMed, SCOPUS, Embase, and Cochrane Central) were screened from inception to October 5, 2020. The following search strategy was used in screening for relevant studies: (galcanezumab OR emgality OR LY2951742 OR LY-2951742 OR ajovy OR galcanezumab-gnlm OR aimovig) AND (migraine). There was no language restriction.

\section{Eligibility criteria}

Studies meeting the following criteria were included: (i) Patients: individuals with migraine, (ii) Intervention: galcanezumab at a dose of $120 \mathrm{mg}$ or $240 \mathrm{mg}$, (iii) Comparator: placebo, (iv) Outcomes: prespecified efficacy (primary) and safety (secondary) outcomes, and (v) Study design: randomized placebocontrolled trials. Exclusion criteria included (i) patients with conditions other than migraine, (ii) interventional monoclonal antibodies other than galcanezumab, (iii) doses of galcanezumab other than 120 mg or $240 \mathrm{mg}$, (iv) animal trials, (v) nonrandomized human clinical trials, and (vi) studies not reporting the prespecified efficacy or safety outcomes.

\section{Study selection}

After screening of studies, duplicates were removed, and the remaining studies underwent a two-stage screening process. The first stage involved title and abstract screening. The second stage involved conducting full-text screening to exclude irrelevant trials. Moreover, we manually searched the reference lists of included studies to consider additional relevant studies. Two authors independently screened the studies and conflicts were resolved by a third author.

\section{Risk of bias assessment}

Cochrane Collaboration's risk of bias tool was used to assess the risk of bias of the included randomized placebo-controlled trials [9]. This risk tool consists of six domains: (i) sequence generation, (ii) allocation concealment, (iii) outcomes blinding, (iv) incomplete data, (v) selective reporting, and (vi) other bias. We scored each domain as unclear, low, or high risk. Two authors independently assessed the risk of bias, and conflicts were resolved by a third author.

\section{Data extraction}

The following three categories of data were collected: (i) baseline characteristics of the included studies, (ii) efficacy outcomes, and (iii) safety outcomes. Baseline characteristics of the included studies included first author, year of publication, national clinical trial (NCT) identifier, phase of clinical trial, type of migraine, study group, and sample size. Efficacy outcomes included change in monthly migraine headache days (MHDs), change in monthly MHDs with acute medication use, patient global impression of severity (PGI-S) score, migraine-specific quality of life role function-restrictive (MSQ RF-R) domain score, and migraine disability assessment (MIDAS) score. Safety outcomes included frequency of injection-site pain, nasopharyngitis, and upper respiratory tract infection (URTI). Several authors extracted the necessary data.

\section{Data analysis}

Review Manager Software Version 5.4.1 was used for statistical analysis. Mean difference (MD) and risk ratio 
(RR) with 95\% confidence interval $(95 \% \mathrm{CI})$ were used to analyze continuous and dichotomous outcomes, respectively. Fixed-effects and random-effects models were used to analyze homogenous and heterogeneous data, respectively. Statistical heterogeneity between studies was assessed by I-squared $\left(\mathrm{I}^{2}\right)$ test and the pvalue of heterogeneity. Statistical heterogeneity was determined when $\mathrm{I}^{2}$ measured $>50 \%$ and $\mathrm{p}$-value of heterogeneity measured $<0.1$. Sensitivity analysis using Cochrane's leave-one-out method was used to resolve heterogonous outcomes. For all outcomes, subgroup analysis according to the galcanezumab dose was conducted (120 mg/240 mg versus placebo).

\section{Results}

\section{Literature search}

Literature search yielded 510 studies. After screening, 490 studies were excluded because they did not match our inclusion criteria. Full-text screening of the remaining 20 studies resulted in an elimination of 14 studies that did not match our inclusion criteria. Finally, six studies comprising 4,023 patients were included in this systematic review and meta-analysis [5,10-14]. Figure 1 shows the PRISMA flowchart.
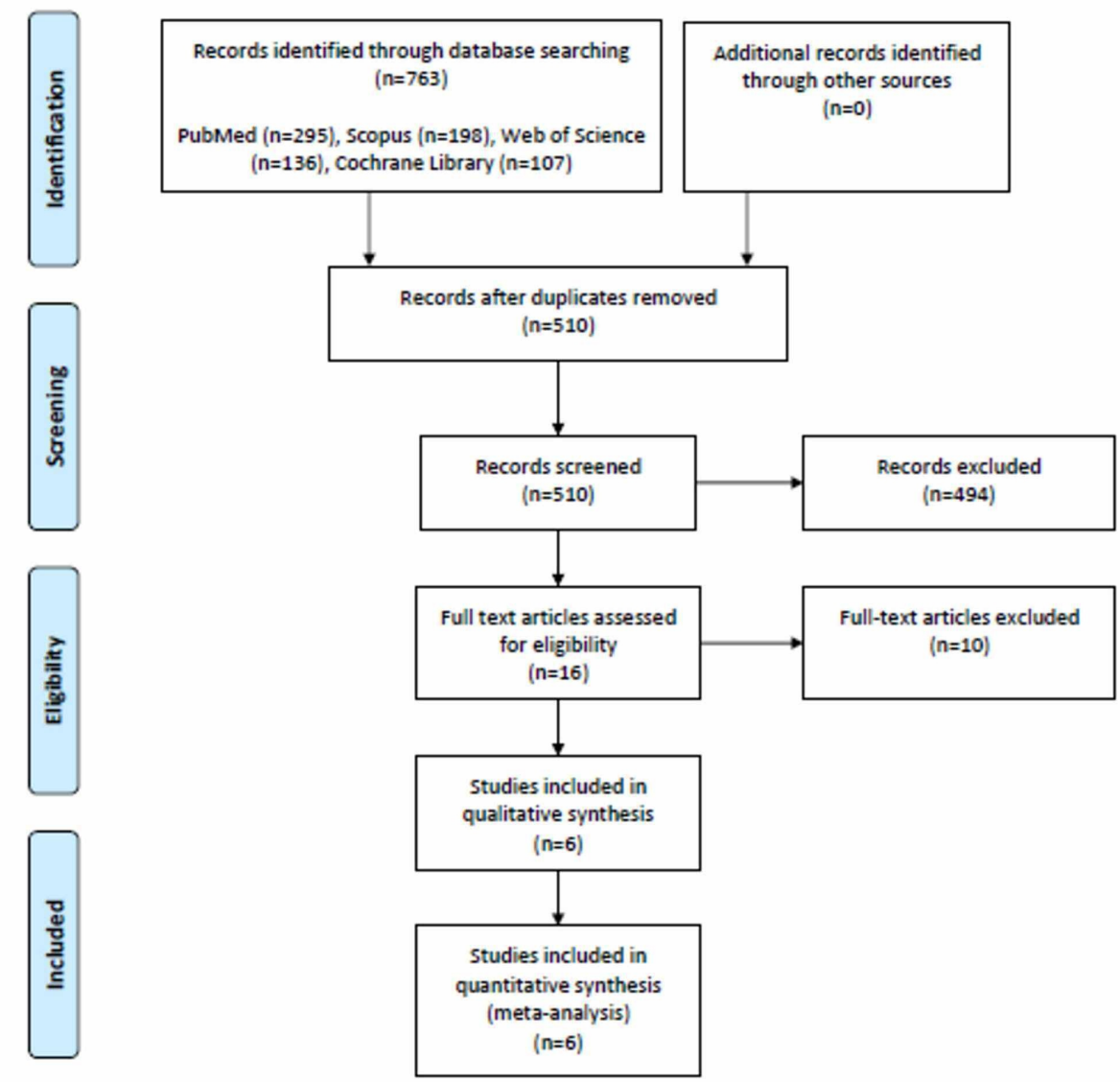

FIGURE 1: PRISMA flowchart.

PRISMA, Preferred Reporting Items for Systematic Reviews and Meta-Analyses

Galcanezumab and placebo were administered to 1,974 and 2,049 patients, respectively. The baseline characteristics of the included studies are depicted in Table 1. 


\section{Cureus}

\begin{tabular}{|c|c|c|c|c|c|c|c|c|c|}
\hline $\begin{array}{l}\text { Study } \\
\text { identifier }\end{array}$ & $\begin{array}{l}\text { NCT } \\
\text { identifier }\end{array}$ & Phase & Condition & Study group & $\mathrm{n}$ & $\begin{array}{l}\text { Female } \\
(\%)\end{array}$ & $\begin{array}{l}\text { Migraine } \\
\text { attacks per } \\
\text { month, mean } \\
\text { (SD) }\end{array}$ & $\begin{array}{l}\text { MHDs per month with } \\
\text { acute medication use, } \\
\text { mean (SD) }\end{array}$ & $\begin{array}{l}\text { MIDAS } \\
\text { baseline } \\
\text { score, mean } \\
\text { (SD) }\end{array}$ \\
\hline \multirow{3}{*}{$\begin{array}{l}\text { Stauffer et al., } \\
2018[5]\end{array}$} & \multirow{3}{*}{ NCT02614183 } & \multirow{3}{*}{3} & \multirow{3}{*}{ Episodic migraine } & $\begin{array}{l}\text { Galcanezumab } \\
(120 \mathrm{mg})\end{array}$ & 213 & 85 & $5.6(1.7)$ & $7.4(3.7)$ & 32.9 (28.2) \\
\hline & & & & $\begin{array}{l}\text { Galcanezumab } \\
(240 \mathrm{mg})\end{array}$ & 212 & 82.6 & $5.7(1.8)$ & $7.3(3.3)$ & $36.1(27.8)$ \\
\hline & & & & Placebo & 433 & 83.6 & $5.8(1.7)$ & $7.4(3.5)$ & $31.8(27.3)$ \\
\hline \multirow{3}{*}{$\begin{array}{l}\text { Detke et al., } \\
2018 \text { [14] }\end{array}$} & \multirow{3}{*}{ NCT02614261 } & \multirow{3}{*}{3} & \multirow{3}{*}{ Chronic migraine } & $\begin{array}{l}\text { Galcanezumab } \\
(120 \mathrm{mg})\end{array}$ & 278 & 85 & NR & $15.1(6.3)$ & $62.5(49.5)$ \\
\hline & & & & $\begin{array}{l}\text { Galcanezumab } \\
(240 \mathrm{mg})\end{array}$ & 277 & 82 & NR & $14.5(6.3)$ & $69.2(64.1)$ \\
\hline & & & & Placebo & 558 & 87 & NR & $15.5(6.6)$ & $68.7(57.4)$ \\
\hline \multirow{2}{*}{$\begin{array}{l}\text { Skljarevski et } \\
\text { al., } 2018 \text { [10] }\end{array}$} & \multirow[t]{2}{*}{ NCT02163993 } & \multirow[t]{2}{*}{2} & \multirow[t]{2}{*}{ Episodic migraine } & $\begin{array}{l}\text { Galcanezumab } \\
(120 \mathrm{mg})\end{array}$ & 70 & 84.6 & $4.6(1.6)$ & NR & NR \\
\hline & & & & Placebo & 137 & 79.6 & $4.7(1.5)$ & NR & NR \\
\hline \multirow{3}{*}{$\begin{array}{l}\text { Skljarevski et } \\
\text { al., } 2018 \text { [11] }\end{array}$} & \multirow{3}{*}{ NCT02614196 } & \multirow{3}{*}{3} & \multirow{3}{*}{ Episodic migraine } & $\begin{array}{l}\text { Galcanezumab } \\
(120 \mathrm{mg})\end{array}$ & 231 & 85.3 & $5.54(1.8)$ & $7.47(3.3)$ & $30.9(27.9)$ \\
\hline & & & & $\begin{array}{l}\text { Galcanezumab } \\
(240 \mathrm{mg})\end{array}$ & 231 & 85.7 & $5.66(1.8)$ & $7.47(3.3)$ & $32.8(28.8)$ \\
\hline & & & & Placebo & 461 & 85.3 & $5.7(1.8)$ & $7.6(3.4)$ & $34.3(31.0)$ \\
\hline \multirow{3}{*}{$\begin{array}{l}\text { Sakai et al., } \\
2020 \text { [12] }\end{array}$} & \multirow{3}{*}{ NCT02959177 } & \multirow{3}{*}{2} & \multirow{3}{*}{ Episodic migraine } & $\begin{array}{l}\text { Galcanezumab } \\
(120 \mathrm{mg})\end{array}$ & 115 & 82.6 & $5.6(1.7)$ & $7.3(2.9)$ & $14.8(18.1)$ \\
\hline & & & & $\begin{array}{l}\text { Galcanezumab } \\
(240 \mathrm{mg})\end{array}$ & 114 & 84.2 & $5.5(1.8)$ & $7.8(3.0)$ & $13.7(13.9)$ \\
\hline & & & & Placebo & 230 & 85.2 & $5.5(1.7)$ & $7.4(3.0)$ & $15.8(19.3)$ \\
\hline \multirow{2}{*}{$\begin{array}{l}\text { Mulleners et } \\
\text { al., } 2020 \text { [13] }\end{array}$} & \multirow[t]{2}{*}{ NCT03559257 } & \multirow[t]{2}{*}{3} & \multirow{2}{*}{$\begin{array}{l}\text { Episodic migraine } \\
\text { and chronic } \\
\text { migraine }\end{array}$} & $\begin{array}{l}\text { Galcanezumab } \\
(120 \mathrm{mg})\end{array}$ & 232 & 84 & NR & $12.3(6)$ & $50.9(46)$ \\
\hline & & & & Placebo & 230 & 88 & NR & $12.4(6)$ & $51(45.5)$ \\
\hline
\end{tabular}

\section{TABLE 1: Baseline characteristics of the included studies.}

MHD, monthly headache days; MIDAS, migraine disability assessment; NCT, national clinical trial; NR, not reported; SD, standard deviation

\section{Results of risk of bias assessment}

All studies showed low risk of bias for the domains of random sequence generation, blinding of participants and personnel, incomplete outcome data, and selective reporting. Inadequate details were provided for allocation concealment and blinding of outcome assessments in some studies, and hence these domains were scored as unclear risk. Overall, all included studies revealed low-to-moderate risk of bias. Figure 2 shows the risk of bias summary and graph. 


\section{Cureus}
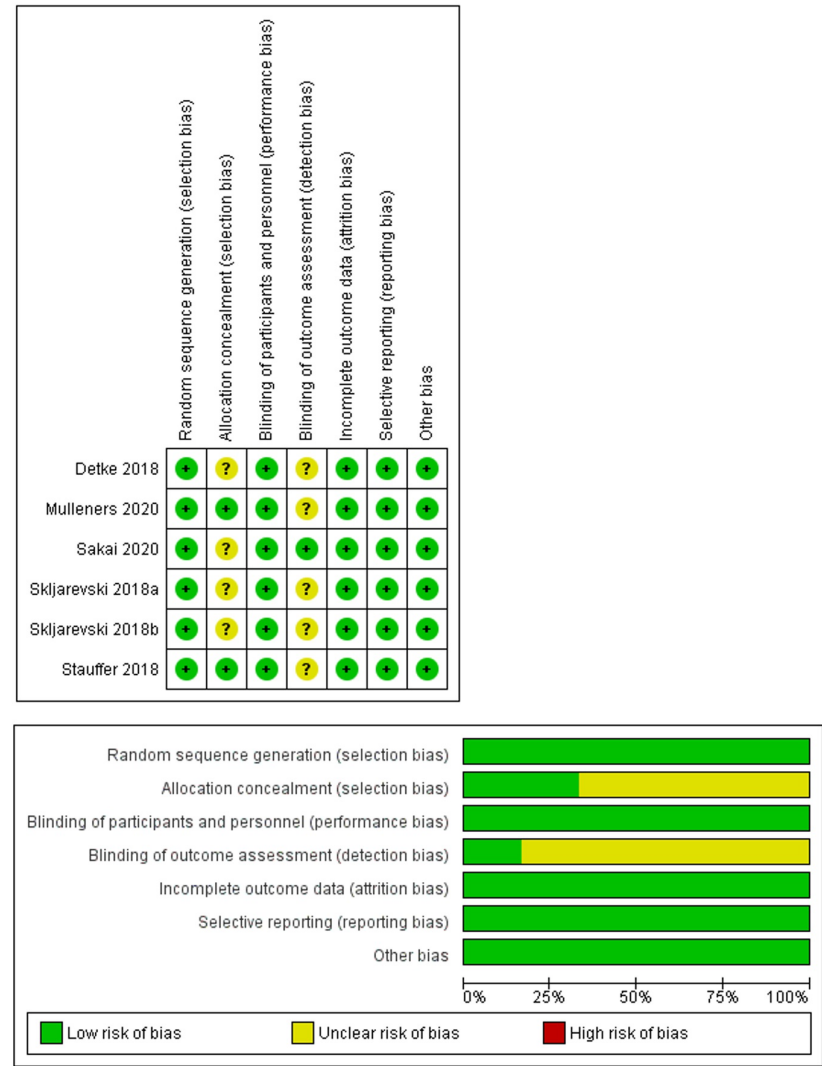

FIGURE 2: Risk of bias summary and graph.

Efficacy outcome: overall mean change from baseline in the number of monthly MHDs

The overall effect size significantly favored galcanezumab over placebo (MD=2.28; $95 \%$ CI [2.02, 2.55]; $\mathrm{p}<0.001)$. Pooled results were homogeneous $\left(\mathrm{I}^{2}=34 \% ; \mathrm{p}=0.15\right)$, and the fixed-effects model was used (Figure 3). Subgroup analysis was performed according to the galcanezumab dose. For galcanezumab $120 \mathrm{mg}$ versus placebo, the overall effect size significantly favored the galcanezumab group ( $\mathrm{MD}=2.39$; $95 \% \mathrm{CI}$ : [2.04, 2.74]; $p<0.001)$. Pooled results were homogeneous $\left(I^{2}=46 \% ; p=0.11\right)$. For galcanezumab $240 \mathrm{mg}$ versus placebo, the overall effect size significantly favored the galcanezumab group ( $\mathrm{MD}=2.14 ; 95 \% \mathrm{CI}[1.73,2.55]$; $\mathrm{p}<0.001)$. Pooled results were homogeneous $\left(\mathrm{I}^{2}=22 \% ; \mathrm{p}=0.28\right)$.

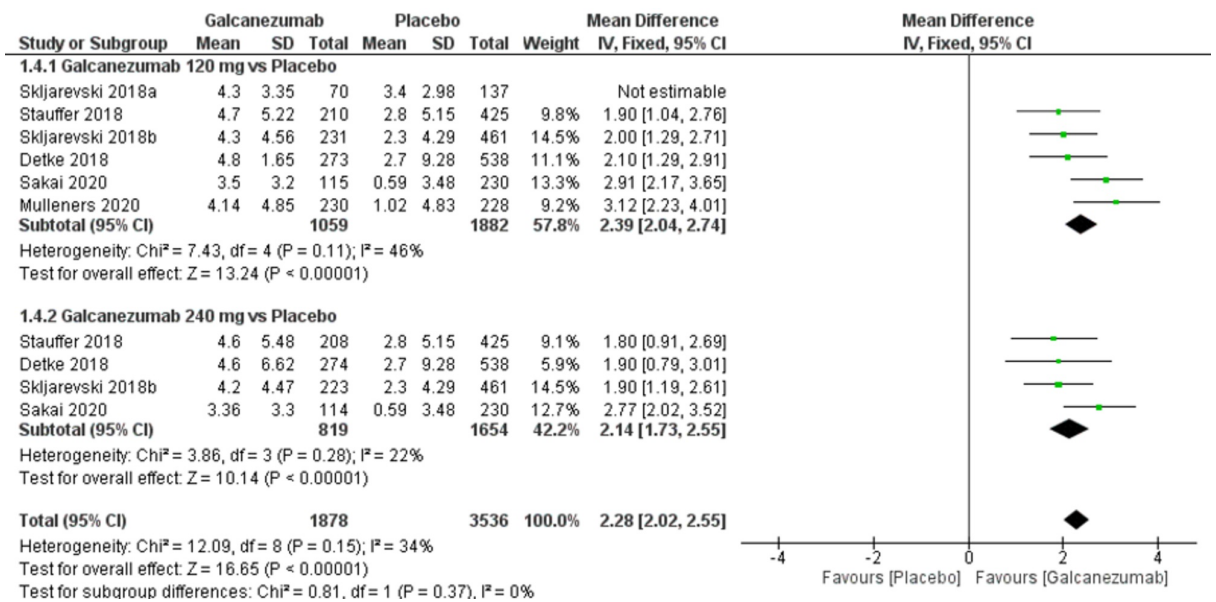

FIGURE 3: Forest plot showing the change in monthly migraine headache days between galcanezumab and placebo groups. 


\section{Efficacy outcome: overall mean change from baseline in the number of monthly MHDs with acute medication use}

The overall effect size significantly favored galcanezumab over placebo (MD=2.22; 95\% CI [1.82, 2.63]; $\mathrm{p}<0.001)$. Pooled results were heterogeneous $\left(\mathrm{I}^{2}=60 \% ; \mathrm{p}=0.010\right)$, and the random-effects model was used (Figure 4). Subgroup analysis was performed according to the galcanezumab dose. For galcanezumab $120 \mathrm{mg}$ versus placebo, the overall effect size significantly favored the galcanezumab group ( $\mathrm{MD}=2.44$; $95 \% \mathrm{CI}$ [1.81, 3.06]; $\mathrm{p}<0.001)$. Pooled results were heterogeneous $\left(\mathrm{I}^{2}=69 \% ; \mathrm{p}=0.01\right)$. Heterogeneity was best resolved $\left(\mathrm{I}^{2}=50 \% ; \mathrm{p}=0.11\right)$ by omitting Mulleners et al.' study [13], and the overall effect size still significantly favored the galcanezumab group ( $\mathrm{MD}=2.19 ; 95 \% \mathrm{CI}[1.65,2.73]$; $\mathrm{p}<0.001$ ). For galcanezumab $240 \mathrm{mg}$ versus placebo, the overall effect size significantly favored the galcanezumab group ( $\mathrm{MD}=1.97 ; 95 \% \mathrm{CI}[1.49,2.44] ; \mathrm{p}<0.001$ ). Pooled results were homogeneous $\left(\mathrm{I}^{2}=39 \% ; \mathrm{p}=0.18\right)$.

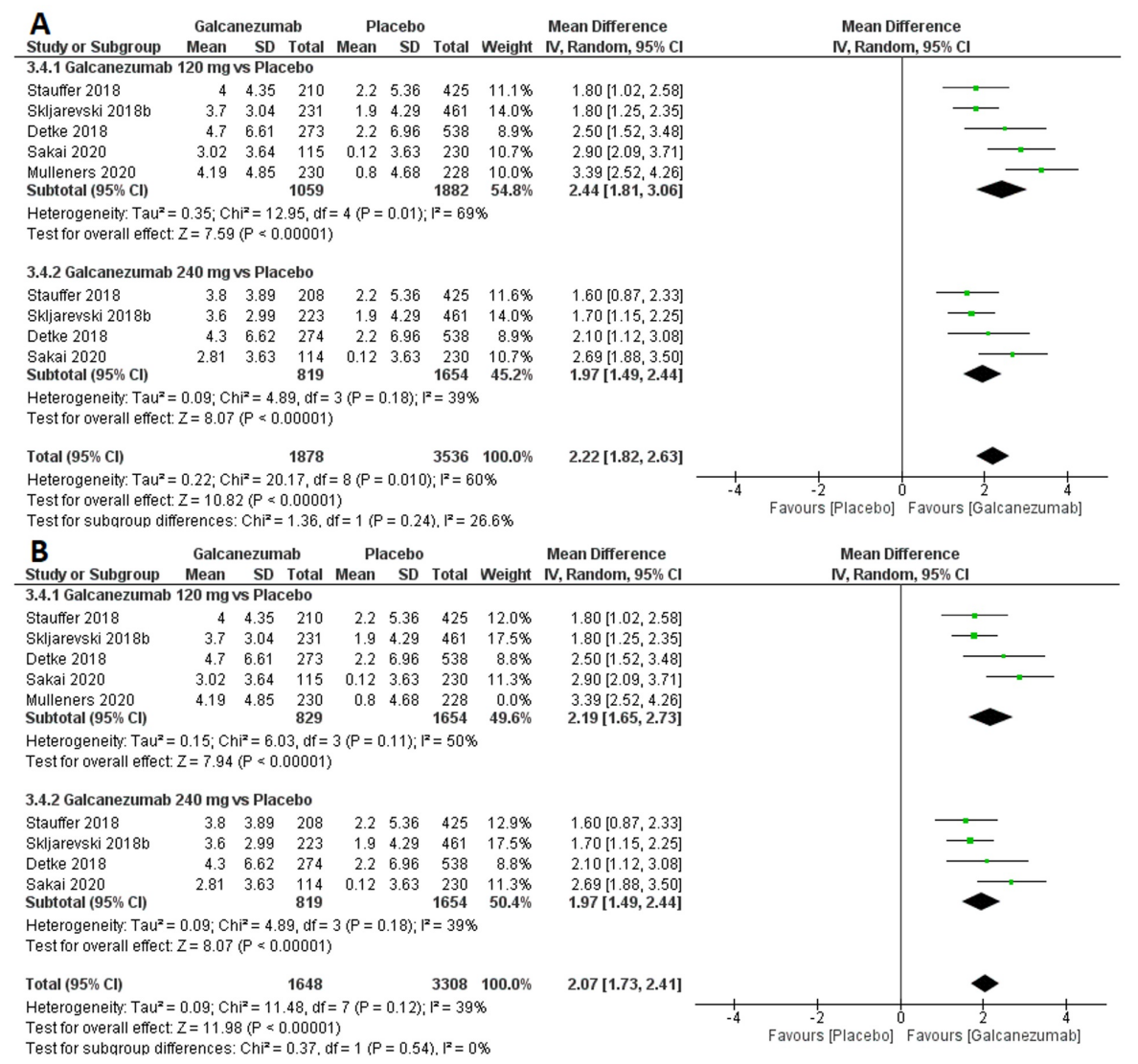

\section{FIGURE 4: Forest plot showing the change in monthly migraine headache days with acute medication use between galcanezumab and placebo groups before (A) and after (B) sensitivity analysis using the leave-one-out method.}

\section{Efficacy outcome: PGI-S score}

The overall effect size significantly favored galcanezumab over placebo (MD $=0.26 ; 95 \% \mathrm{CI}[0.18,0.34]$; $\mathrm{p}<0.001)$. Pooled results were homogenous $\left(\mathrm{I}^{2}=0 \% ; \mathrm{p}=0.84\right)$, and the fixed-effects model was used (Figure 5). Subgroup analysis was performed according to the galcanezumab dose. For galcanezumab $120 \mathrm{mg}$ versus placebo, the overall effect size significantly favored the galcanezumab group ( $\mathrm{MD}=0.24 ; 95 \% \mathrm{CI}[0.13,0.35]$; $\mathrm{p}<0.001)$. Pooled results were homogeneous $\left(\mathrm{I}^{2}=0 \% ; \mathrm{p}=0.44\right)$. For galcanezumab $240 \mathrm{mg}$ versus placebo, the overall effect size significantly favored the galcanezumab group $(0.28[0.16,0.41] ; \mathrm{p}<0.001)$. Pooled results were homogeneous $\left(\mathrm{I}^{2}=0 \%\right.$; $\left.\mathrm{p}=0.99\right)$. 


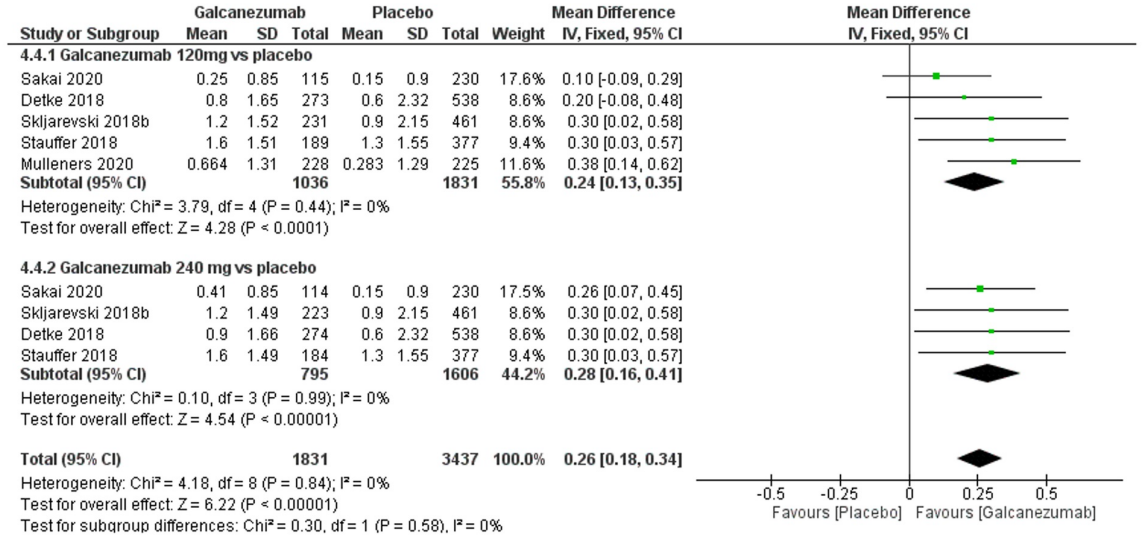

FIGURE 5: Forest plot showing PGI-S between galcanezumab and placebo groups.

PGI-S, global impression score of severity

\section{Efficacy outcome: MSQ RF-R score}

The overall effect size significantly favored galcanezumab over placebo $(\mathrm{MD}=4.39 ; 95 \% \mathrm{CI}[2.10,6.68]$; $\mathrm{p}<0.001)$. Pooled results were heterogeneous $\left(\mathrm{I}^{2}=72 \%\right.$; $\left.<0.001\right)$, and the random-effects model was used (Figure 6). Subgroup analysis was performed according to the galcanezumab dose. For galcanezumab $120 \mathrm{mg}$ versus placebo, the overall effect size did not significantly differ between both groups ( $\mathrm{MD}=2.06$; $95 \%$ CI [$1.79,5.90] ; \mathrm{p}=0.32)$. Pooled results were heterogeneous $\left(\mathrm{I}^{2}=80 \% ; \mathrm{p}=0.002\right)$. Heterogeneity was best resolved $\left(\mathrm{I}^{2}=0 \% ; \mathrm{p}=0.53\right)$ by omitting Sakai et al.'s study [12], and the overall effect size still did not favor any group $(\mathrm{MD}=0.37 ; 95 \% \mathrm{CI}[-1.75,2.50] ; \mathrm{p}=0.73)$. For galcanezumab $240 \mathrm{mg}$ versus placebo, the overall effect size significantly favored the galcanezumab group ( $\mathrm{MD}=6.59 ; 95 \% \mathrm{CI}[4.92,8.26] ; \mathrm{p}<0.001)$. Pooled results were homogeneous $\left(\mathrm{I}^{2}=0 \%\right.$; $\left.\mathrm{p}=0.88\right)$.

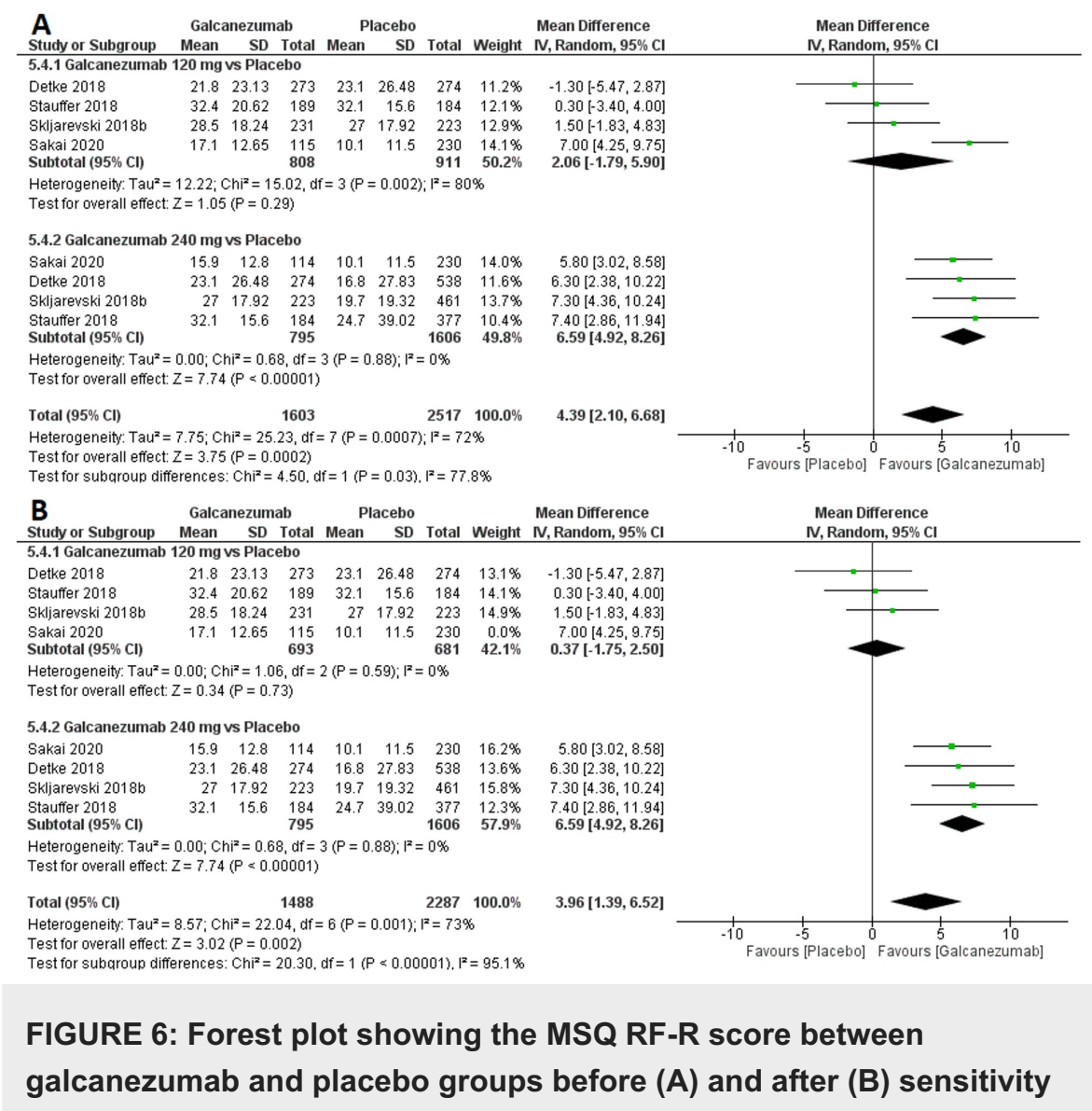


analysis using the leave-one-out method.

MSQ RF-R, migraine-specific quality of life questionnaire role function-restrictive

\section{Efficacy outcome: MIDAS score}

The overall effect size significantly favored galcanezumab over placebo (MD=6.83; 95\% CI [1.35, 12.32]; $\mathrm{p}<0.001)$. Pooled results were heterogeneous $\left(\mathrm{I}^{2}=69 \% ; \mathrm{p}=0.01\right)$, and the random-effects model was used (Figure 7). Subgroup analysis was performed according to the galcanezumab dose. For galcanezumab $120 \mathrm{mg}$ versus placebo, the overall effect size significantly favored the galcanezumab group (MD $=7.06 ; 95 \% \mathrm{CI}[-3.68$, 17.81]; $\mathrm{p}=0.20$ ). Pooled results were heterogeneous $\left(\mathrm{I}^{2}=81 \% ; \mathrm{p}=0.005\right)$. Heterogeneity was best resolved $\left(\mathrm{I}^{2}=0 \% ; \mathrm{p}=0.71\right)$ by omitting Mulleners et al.'s study [13], and the overall effect size did not favor any group $(\mathrm{MD}=1.29 ; 95 \% \mathrm{CI}[-2.76,5.35] ; \mathrm{p}=0.53)$. For galcanezumab $240 \mathrm{mg}$ versus placebo, the overall effect size significantly favored the galcanezumab group ( $\mathrm{MD}=7.85 ; 95 \% \mathrm{CI}[4.08,11.62]$; $\mathrm{p}<0.001)$. Pooled results were homogeneous $\left(\mathrm{I}^{2}=0 \%\right.$; $\left.\mathrm{p}=0.64\right)$.

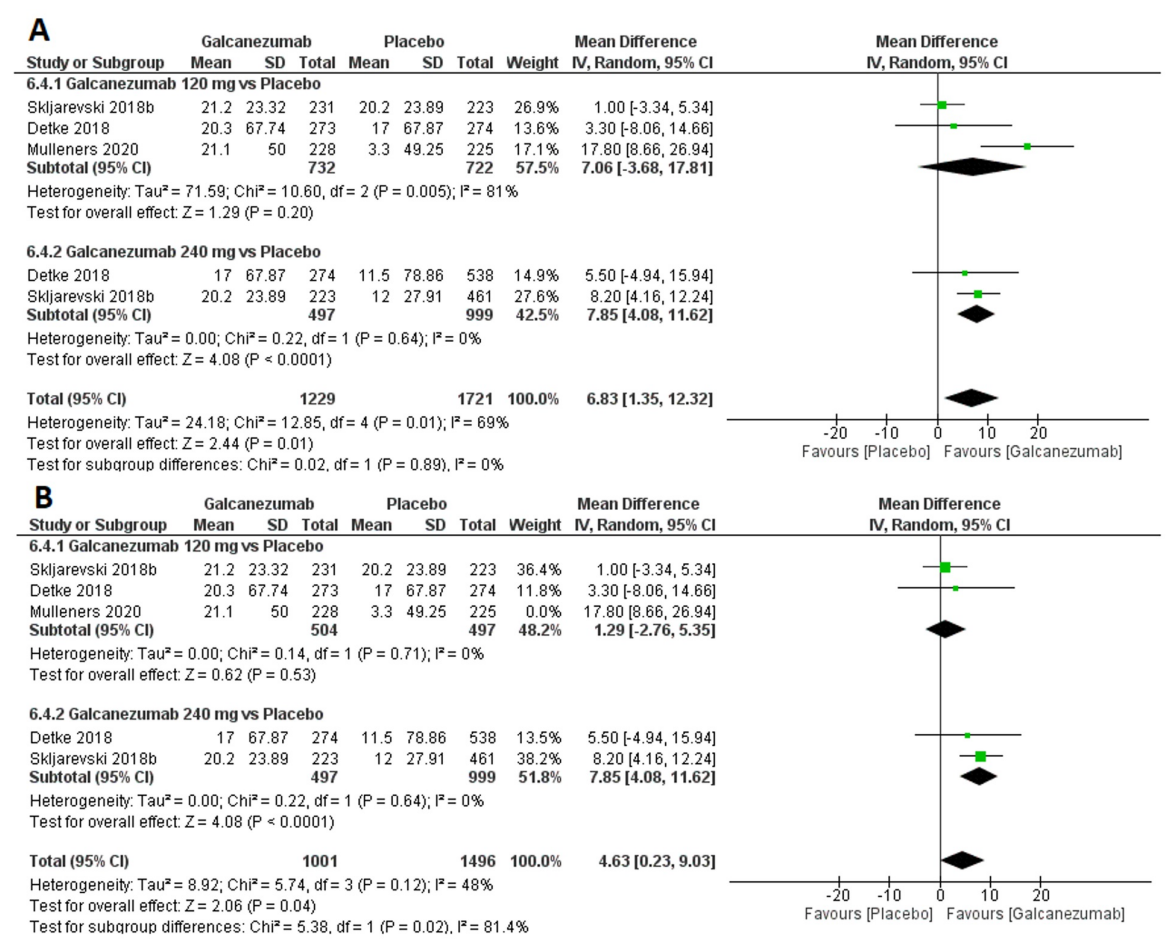

\section{FIGURE 7: Forest plot showing MIDAS between galcanezumab and placebo groups before (A) and after (B) sensitivity analysis using the leave-one-out method.}

MIDAS, migraine disability assessment score

\section{Safety outcome: injection-site pain}

The overall effect size did not show a significant difference between both groups ( $R R=1.35$; $95 \%$ CI $[0.98$, 1.86]; $\mathrm{p}=0.06)$ Pooled results were heterogeneous $\left(\mathrm{I}^{2}=62 \% ; \mathrm{p}=0.005\right)$, and the random-effects model was used (Figure 8). Subgroup analysis was performed according to the galcanezumab dose. For galcanezumab $120 \mathrm{mg}$ versus placebo, the overall effect size significantly did not differ between both groups ( $R R=1.34 ; 95 \% \mathrm{CI}$ $[0.79,2.27] ; \mathrm{p}=0.28)$. Pooled results were heterogeneous $\left(\mathrm{I}^{2}=71 \% ; \mathrm{p}=0.004\right)$. Heterogeneity could not be resolved by performing leave-one-out method. For galcanezumab $240 \mathrm{mg}$ versus placebo, the overall effect size did not significantly differ between both groups $(\mathrm{RR}=1.40 ; 95 \% \mathrm{CI}[0.93,2.11] ; \mathrm{p}=0.11)$. Pooled results were homogeneous $\left(\mathrm{I}^{2}=52 \%\right.$; $\left.\mathrm{p}=0.10\right)$. 


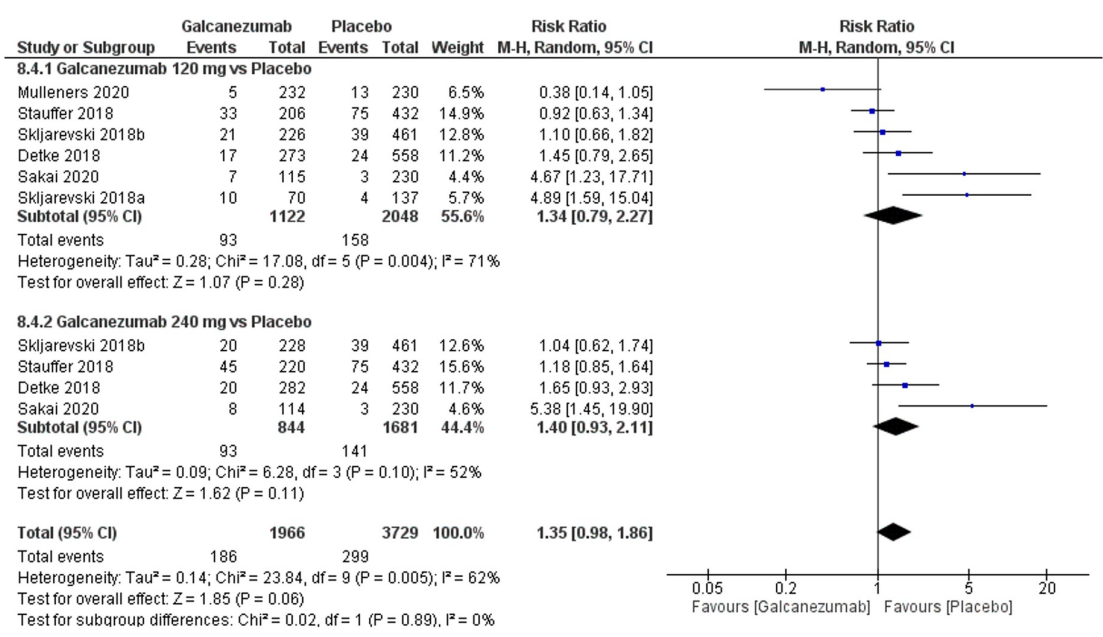

FIGURE 8: Forest plot showing the rate of injection-site pain between galcanezumab and placebo groups.

\section{Safety outcome: nasopharyngitis}

The overall effect size did not show a significant difference between both groups (MD $=0.93 ; 95 \% \mathrm{CI}[0.74$, $1.16] ; \mathrm{p}=0.5)$. Pooled results were homogenous $\left(\mathrm{I}^{2}=35 \% ; \mathrm{p}=0.15\right)$, and the fixed-effects model was used (Figure 9). Subgroup analysis was performed according to the galcanezumab dose. For galcanezumab $120 \mathrm{mg}$ versus placebo, the overall effect size did not significantly differ between both groups ( $\mathrm{RR}=1.11$; $95 \% \mathrm{CI}$ $[0.84,1.47] ; \mathrm{p}=0.47)$. Pooled results were homogeneous $\left(\mathrm{I}^{2}=29 \% ; \mathrm{p}=0.23\right)$. For galcanezumab $240 \mathrm{mg}$ versus placebo, the overall effect size did not significantly differ between both groups ( $R R=0.68 ; 95 \% \mathrm{CI}[0.46,1.00]$; $\mathrm{p}=0.05)$. Pooled results were homogeneous $\left(\mathrm{I}^{2}=53 \%\right.$; $\left.\mathrm{p}=0.53\right)$.

\begin{tabular}{|c|c|c|c|c|c|c|}
\hline \multirow[b]{2}{*}{ Study or Subgroup } & \multicolumn{2}{|c|}{ Galcanezumab } & \multicolumn{2}{|c|}{ Placebo } & \multirow[b]{2}{*}{ Weight } & \multirow{2}{*}{$\begin{array}{l}\text { Risk Ratio } \\
\text { M-H, Fixed, } 95 \% \mathrm{Cl}\end{array}$} \\
\hline & & Total & & & & \\
\hline \multicolumn{7}{|c|}{ 9.4.1 Galcanezumab $120 \mathrm{mg}$ vs Placebo } \\
\hline Mulleners 2020 & 16 & 232 & 21 & 230 & $14.3 \%$ & $0.76[0.40,1.4$ \\
\hline Skljarevski $2018 \mathrm{~b}$ & 19 & 226 & 41 & 461 & $18.3 \%$ & $0.95[0.56,1.59]$ \\
\hline Stauffer 2018 & 16 & 206 & 27 & 432 & $11.8 \%$ & $1.24[0.68,2.25]$ \\
\hline Detke 2018 & 17 & 273 & 26 & 558 & $11.6 \%$ & $1.34[0.74,2.42]$ \\
\hline & 6 & & 3 & & & \\
\hline Subtotal $\left(95^{\circ}\right.$ & & 1007 & & 1818 & $57.4 \%$ & \\
\hline \multirow{2}{*}{\multicolumn{7}{|c|}{ Heterogeneity: $\mathrm{Chi}^{2}=5.66, \mathrm{df}=4(\mathrm{P}=0.23) ; \mathrm{I}^{2}=29 \%$}} \\
\hline & & & & & & \\
\hline \multicolumn{7}{|c|}{ 9.4.2 Galcanezumab $240 \mathrm{mg}$ vs placebo } \\
\hline Stauffer 2018 & 6 & 220 & 27 & 432 & $12.4 \%$ & $0.44[0$ \\
\hline Detke & 9 & & 26 & 558 & & \\
\hline & 17 & 228 & 41 & 461 & 18.4 & \\
\hline Subtotal $(95 \% \mathrm{Cl})$ & & 730 & & 1451 & $42.6 \%$ & $0.68[0.46,1.0$ \\
\hline \multirow{3}{*}{\multicolumn{7}{|c|}{$\begin{array}{l}\text { Heterogeneity: } \mathrm{Chi}^{2}=1.57, \mathrm{df}=2(\mathrm{P}=0.46) ; \mathrm{I}^{2}=0 \% \\
\text { Test for overall effect: } Z=1.95(\mathrm{P}=0.05)\end{array}$}} \\
\hline & & & & & & \\
\hline & & & & & & \\
\hline Total $(95 \% \mathrm{Cl})$ & & 1737 & & 3269 & $100.0 \%$ & $0.93[0.74,1.16$ \\
\hline & 106 & & & & & \\
\hline \multirow{2}{*}{\multicolumn{7}{|c|}{$\begin{array}{l}\text { Heterogeneity: Chi } \mathrm{i}^{2}=10.80, \mathrm{df}=7(P=0.15) ; \mathrm{I}^{2}=35 \% \\
\text { Test for overall effect: } Z=0.67(P=0.50)\end{array}$}} \\
\hline & & & & & & \\
\hline & & & & & & \\
\hline
\end{tabular}

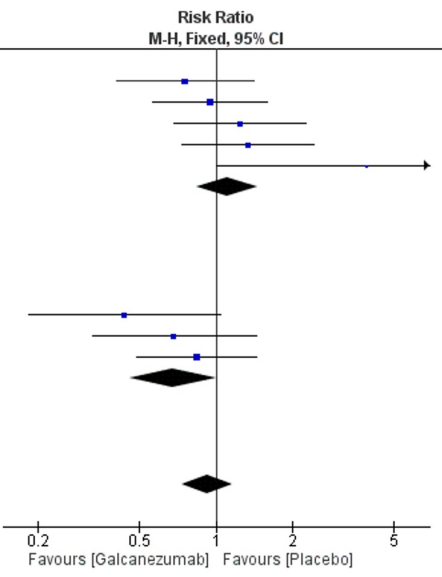

FIGURE 9: Forest plot showing the rate of nasopharyngitis between galcanezumab and placebo groups.

\section{Safety outcome: URTI}

The overall effect size significantly showed a significantly higher frequency of URTI in the galcanezumab group ( $R R=1.61 ; 95 \%$ CI $[1.16,2.24] ; \mathrm{p}=0.004)$. Pooled results were homogenous $\left(\mathrm{I}^{2}=0 \%\right.$; $\left.\mathrm{p}=0.53\right)$, and the fixed-effects model was used (Figure 10). Subgroup analysis was performed according to the galcanezumab dose. For galcanezumab $120 \mathrm{mg}$ versus placebo, the overall effect size revealed a significantly higher occurrence of URTI in the galcanezumab group ( $\mathrm{RR}=1.79 ; 95 \% \mathrm{CI}[1.17,2.72]$; $\mathrm{p}=0.007)$. Pooled results were homogeneous $\left(\mathrm{I}^{2}=30 \%\right.$; $\left.\mathrm{p}=0.23\right)$. For galcanezumab $240 \mathrm{mg}$ versus placebo, the overall effect size did not differ between both groups $(\mathrm{RR}=1.38 ; 95 \% \mathrm{CI}[0.81,2.35]$; $\mathrm{p}=0.24)$. Pooled results were homogeneous $\left(\mathrm{I}^{2}=0 \%\right.$; $\mathrm{p}=0.80$ ). 


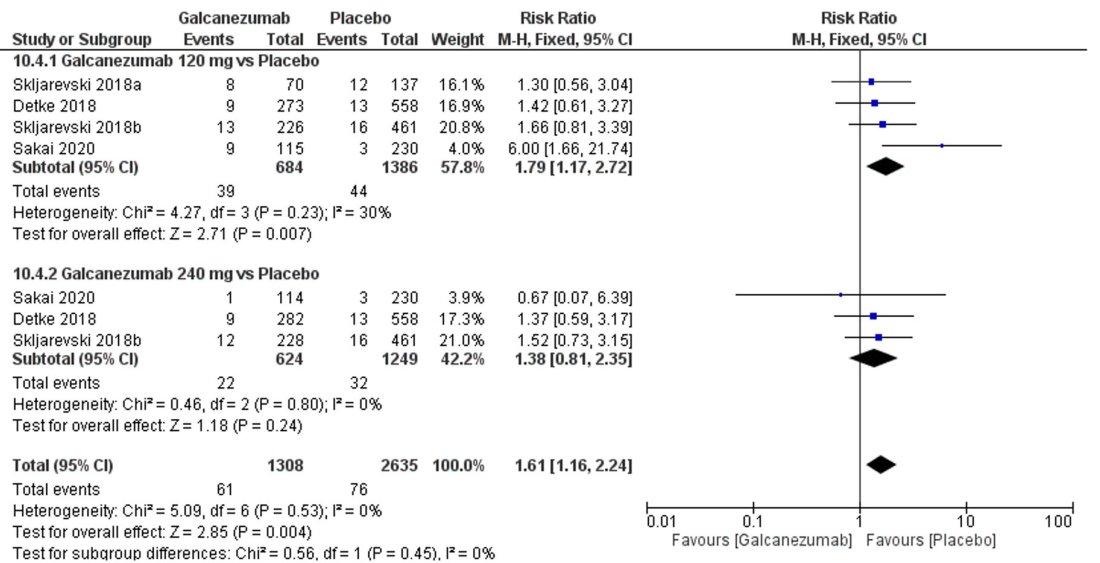

\section{FIGURE 10: Forest plot showing the rate of upper respiratory tract infection between galcanezumab and placebo groups.}

\section{Discussion}

Our analysis found that galcanezumab was highly effective in the management of migraine attacks. Specifically, galcanezumab succeeded in decreasing monthly MHDs and monthly MHDs with acute medication use. Overall, when compared to placebo, our results revealed that both doses of galcanezumab provided nearly equal therapeutic efficacy for most outcomes, except for MSQ RF-R and MIDAS scores where galcanezumab $240 \mathrm{mg}$ showed a significantly higher efficacy when compared with galcanezumab $120 \mathrm{mg}$. With regard to side effects, the rates of injection-site pain and nasopharyngitis did not substantially differ between galcanezumab (inclusive of $120 \mathrm{mg}$ and $240 \mathrm{mg}$ ) and placebo groups. Nonetheless, when compared to placebo, galcanezumab $120 \mathrm{mg}$, but not galcanezumab $240 \mathrm{mg}$, substantially correlated with a higher rate of URTI.

The favorable efficacy of galcanezumab for the management of migraine is somehow anticipated, as galcanezumab has been depicted to be effective in managing other neurological disorders. In a recent review, galcanezumab has demonstrated promising results for both prevention and treatment of cluster headache $[6,15,16]$. Nonetheless, when administered to patients with osteoarthritis, galcanezumab failed to reduce signs and symptoms in patients with knee osteoarthritis [17].

Similar drugs of the same anti-CGRP monoclonal antibodies have been previously tried for migraine and reported encouraging results. For example, erenumab proved to be effective in the prevention and treatment of migraine [18]. A systematic review and meta-analysis of five randomized placebo-controlled trials revealed the superiority of erenumab over placebo in reducing the monthly MHDs and migraine-specific medication days [19]. Fremanezumab is a humanized monoclonal antibody that targets the CGRP receptor. Fremanezumab showed promising results in the treatment and prevention of migraine, with a very low incidence of side effects [20]. Both fremanezumab and erenumab could advantageously convert patients from chronic migraine status to episodic migraine status [21]. No trials till now have yet compared fremanezumab and galcanezumab to determine which drug is more effective and safer.

Generally, common side effects of anti-CGRP monoclonal antibodies include URTI, nasopharyngitis, urinary tract infection, and injection-site pain. Deng et al. [22] conducted a meta-analysis of 11 randomized placebo-controlled trials comparing anti-CGRP monoclonal antibodies versus placebo. The authors revealed that galcanezumab, fremanezumab, and erenumab significantly resulted in reduction of MHDs and acute migraine-specific medication days, in addition to an enhancement in $50 \%$ responder rate. Moreover, the adverse events and treatment discontinuation frequencies secondary to adverse events were not considerably dissimilar between the anti-CGRP monoclonal antibodies and placebo groups. In subgroup analysis, comparable efficacy and tolerability outcomes were achieved for galcanezumab, fremanezumab, and erenumab. Similar findings were reciprocated in other meta-analyses by Zhu et al. [23] and Xu et al. [24]. In the literature, various doses of galcanezumab have been used, ranging from as low as $5 \mathrm{mg}$ to as high as $300 \mathrm{mg}$. The optimal dose that yields maximum efficacy and minimum adverse events is yet to be determined.

Our study has several strengths. The large number of included trials is the main strength of our study when compared to previous meta-analysis studies [25-27]. We only included randomized placebo-controlled clinical trials to ensure high-quality evidence. Moreover, we performed subgroup analysis according to the two most commonly used galcanezumab doses $(120 \mathrm{mg}$ and $240 \mathrm{mg}$ ) and excluded the others to ensure consistency with regard to drug dosing. Whenever heterogeneity existed during meta-analysis, we used the 
leave-one-out method to resolve the heterogeneity. Nonetheless, our study is not without limitations. The vast majority of studies had an unclear risk of bias regarding two important domains: allocation concealment and blinding of outcome assessment. This observation could negatively impact the quality of the evaluated outcomes. Moreover, some of the reported endpoints revealed significant heterogeneity, which could be ascribed to the varying degrees of migraine severity and duration of treatment. Lastly, not all studies adequately reported our prespecified side effects.

As it stands now, galcanezumab (120 mg and $240 \mathrm{mg}$ ) appears to be clinically safe and effective in the management of patients with migraine. Nonetheless, future research directions should be geared toward determining the optimal dose of galcanezumab for the management of patients with migraine. Moreover, head-to-head comparative studies between galcanezumab and other related anti-CGRP receptor monoclonal antibodies are warranted.

\section{Conclusions}

In summary, this systematic review and meta-analysis examined the efficacy of galcanezumab (120 mg and $240 \mathrm{mg}$ ) versus placebo in patients with migraine. Our findings showed that galcanezumab (120 mg or 240 $\mathrm{mg}$ ) was superior to placebo in reducing the number of MHDs and MHDs with acute medication use. Moreover, galcanezumab treatment significantly correlated with improved PGI-S, MSQ RF-R, and MIDAS scores. Overall, the rates of side effects did not substantially differ between galcanezumab and placebo groups.

\section{Additional Information \\ Disclosures}

Human subjects: All authors have confirmed that this study did not involve human participants or tissue. Animal subjects: All authors have confirmed that this study did not involve animal subjects or tissue. Conflicts of interest: In compliance with the ICMJE uniform disclosure form, all authors declare the following: Payment/services info: All authors have declared that no financial support was received from any organization for the submitted work. Financial relationships: All authors have declared that they have no financial relationships at present or within the previous three years with any organizations that might have an interest in the submitted work. Other relationships: All authors have declared that there are no other relationships or activities that could appear to have influenced the submitted work.

\section{References}

1. Dodick DW: Migraine. Lancet. 2018, 391:1315-1330. 10.1016/s0140-6736(18)30478-1

2. Olesen J: International classification of headache disorders. Lancet Neurol. 2018, 17:396-397. 10.1016/s1474-4422(18)30085-1

3. Lassen LH, Haderslev PA, Jacobsen VB, Iversen HK, Sperling B, Olesen J: CGRP may play a causative role in migraine. Cephalalgia. 2002, 22:54-61. 10.1046/j.1468-2982.2002.00310.x

4. Juhasz G, Zsombok T, Modos EA, et al.: NO-induced migraine attack: strong increase in plasma calcitonin gene-related peptide (CGRP) concentration and negative correlation with platelet serotonin release. Pain. 2003, 106:461-470. 10.1016/i.pain.2003.09.008

5. Stauffer VL, Dodick DW, Zhang Q, Carter JN, Ailani J, Conley RR: Evaluation of galcanezumab for the prevention of episodic migraine: the EVOLVE-1 randomized clinical trial. JAMA Neurol. 2018, 75:10801088. 10.1001/jamaneurol.2018.1212

6. Lamb YN: Galcanezumab: first global approval. Drugs. 2018, 78:1769-1775. 10.1007/s40265-018-1002-7

7. Moher D, Liberati A, Tetzlaff J, Altman DG: Preferred reporting items for systematic reviews and metaanalyses: the PRISMA statement. PLoS Med. 2009, 6:1000097. 10.1371/journal.pmed.1000097

8. Cochrane Collaboration: Cochrane Handbook for Systematic Reviews of Interventions Version 5.1.0 . Higgins JPT, Green S (ed): Cochrane Collaboration, Chichester, UK; 2011.

9. Higgins JP, Altman DG, Gøtzsche PC, et al.: The Cochrane Collaboration's tool for assessing risk of bias in randomised trials. BMJ. 2011, 343:5928. 10.1136/bmj.d5928

10. Skljarevski V, Oakes TM, Zhang Q, et al.: Effect of different doses of galcanezumab vs placebo for episodic migraine prevention: a randomized clinical trial. JAMA Neurol. 2018, 75:187-193. 10.1001/jamaneurol.2017.3859

11. Skljarevski V, Matharu M, Millen BA, Ossipov MH, Kim BK, Yang JY: Efficacy and safety of galcanezumab for the prevention of episodic migraine: results of the EVOLVE-2 Phase 3 randomized controlled clinical trial. Cephalalgia. 2018, 38:1442-1454. 10.1177/0333102418779543

12. Sakai F, Ozeki A, Skljarevski V: Efficacy and safety of galcanezumab for prevention of migraine headache in Japanese patients with episodic migraine: a phase 2 randomized controlled clinical trial. Cephalalgia Rep. 2020, 3:251581632093257. 10.1177/2515816320932573

13. Mulleners WM, Kim BK, Láinez MJA, et al.: Safety and efficacy of galcanezumab in patients for whom previous migraine preventive medication from two to four categories had failed (CONQUER): a multicentre, randomised, double-blind, placebo-controlled, phase 3b trial. Lancet Neurol. 2020, 19:814-825. 10.1016/s1474-4422(20)30279-9

14. Detke HC, Goadsby PJ, Wang S, Friedman DI, Selzler KJ, Aurora SK: Galcanezumab in chronic migraine: the randomized, double-blind, placebo-controlled REGAIN study. Neurology. 2018, 91:2211-2221. 10.1212/wnl.0000000000006640

15. Yuan H, Spare NM, Silberstein SD: Targeting CGRP for the prevention of migraine and cluster headache: a 
narrative review. Headache. 2019, 59:20-32. 10.1111/head.13583

16. Giani L, Proietti Cecchini A, Leone M: Galcanezumab for the prevention of cluster headache . Expert Opin Biol Ther. 2020, 20:1133-1142. 10.1080/14712598.2020.1800635

17. Jin Y, Smith C, Monteith D, et al.: CGRP blockade by galcanezumab was not associated with reductions in signs and symptoms of knee osteoarthritis in a randomized clinical trial. Osteoarthritis Cartilage. 2018, 26:1609-1618. 10.1016/j.joca.2018.08.019

18. Ramón C, Cernuda-Morollón E, Pascual J: Calcitonin gene-related peptide in peripheral blood as a biomarker for migraine. Curr Opin Neurol. 2017, 30:281-286. 10.1097/wco.0000000000000440

19. Zhu C, Guan J, Xiao H, Luo W, Tong R: Erenumab safety and efficacy in migraine: A systematic review and meta-analysis of randomized clinical trials. Medicine (Baltimore). 2019, 98:18483. 10.1097/md.0000000000018483

20. Lionetto L, Curto M, Cisale GY, Capi M, Cipolla F, Guglielmetti M, Martelletti P: Fremanezumab for the preventive treatment of migraine in adults. Expert Rev Clin Pharmacol. 2019, 12:741-748. 10.1080/17512433.2019.1635452

21. Tepper SJ: Anti-calcitonin gene-related peptide (CGRP) therapies: update on a previous review after the American Headache Society 60th Scientific Meeting, San Francisco, June 2018. Headache. 2018, 58:276-290. 10.1111/head.13417

22. Deng H, Li GG, Nie H, Feng YY, Guo GY, Guo WL, Tang ZP: Efficacy and safety of calcitonin-gene-related peptide binding monoclonal antibodies for the preventive treatment of episodic migraine - an updated systematic review and meta-analysis. BMC Neurol. 2020, 20:57. 10.1186/s12883-020-01633-3

23. Zhu Y, Liu Y, Zhao J, Han Q, Liu L, Shen X: The efficacy and safety of calcitonin gene-related peptide monoclonal antibody for episodic migraine: a meta-analysis. Neurol Sci. 2018, 39:2097-2106. 10.1007/s10072-018-3547-3

24. Xu D, Chen D, Zhu LN, Tan G, Wang HJ, Zhang Y, Liu L: Safety and tolerability of calcitonin-gene-related peptide binding monoclonal antibodies for the prevention of episodic migraine - a meta-analysis of randomized controlled trials. Cephalalgia. 2019, 39:1164-1179. 10.1177/0333102419829007

25. Gklinos P, Mitsikostas DD: Galcanezumab in migraine prevention: a systematic review and meta-analysis of randomized controlled trials. Ther Adv Neurol Disord. 2020, 13:1756286420918088. 10.1177/1756286420918088

26. Zhao X, Xu X, Li Q: Efficacy and safety of galcanezumab for preventive treatment of migraine: a systematic review and meta-analysis [Online ahead of print]. J Neurol. 2020, 10.1007/s00415-020-09707-5

27. Yang Y, Wang Z, Gao B, Xuan H, Zhu Y, Chen Z: Different doses of galcanezumab versus placebo in patients with migraine and cluster headache: a meta-analysis of randomized controlled trials. J Headache Pain. 2020, 21:14. 10.1186/s10194-020-1085-x 